\title{
How to be a good reviewer
}

\author{
K. L. Edwards • C. Schizas • A. F. Mannion • \\ M. Aebi $\cdot$ R. Gunzburg
}

Received: 5 December 2014/Published online: 16 December 2014

(C) The Author(s) 2014. This article is published with open access at Springerlink.com

"Peer review is a means of ensuring that the results of scientific research are meaningful and worth reading" J. Weinstein.

"Learn from yesterday, live for today, hope for tomorrow. The important thing is not to stop questioning" Albert Einstein.

Publication of quality research is at the heart of academic and clinical practice. It serves to both further knowledge and promote evidence-based care for patients. One of the ways we seek to improve quality is by peer review. This involves experts in the field critiquing the proposed paper and helping to improve it. The goals of

K. L. Edwards $(\bowtie)$

School of Medicine, University of Nottingham, Nottingham, UK

e-mail: Kimberley.edwards@nottingham.ac.uk

\section{K. L. Edwards}

Arthritis Research UK Centre for Sport, Exercise and Osteoarthritis, Nottingham University Hospitals Trust, Nottingham, UK

C. Schizas

Neuro-orthopaedic Spine Unit, Clinique Cecil, Lausanne, Switzerland

\author{
A. F. Mannion \\ Department of Research and Development, Schulthess Klinik, \\ Zurich, Switzerland \\ M. Aebi \\ "Das Rückenzentrum AG", Hirslanden-Salemspital, \\ 3000 Bern 25, Switzerland \\ R. Gunzburg \\ Cavell Spine Center, Edith Cavell Clinic, Brussels, Belgium
}

peer review are twofold: to ensure the accuracy and improve the quality and transparency of a paper through constructive criticism; and to ensure that clinical decisions about patient care are made on the basis of sound research evidence. At the recent EuroSpine conference in Lyon, we ran a training workshop to guide reviewers in the review process.

The session started with an overview of the ethics of reviewing papers by Dr. Robert Gunzburg, the Editor-inChief of European Spine Journal. The review process was discussed and data showing that papers that are published in the second choice of journal (after having received comments from a first choice journal that did not accept it for publication) are cited more frequently than those accepted by the first journal; this suggests that the process of review, rejection, and revision helps to improve the quality of the reports and the accessibility of the science. A clear ethical issue is that of the need for both authors and reviewers to disclose both financial and non-financial conflicts of interest, such as intellectual passion, relationships or affiliations. He also reminded us that good doctors use both individual clinical expertise and the best available external evidence to steer their practice, and that neither alone is sufficient. Without clinical expertise, practice risks becoming tyrannised by evidence, because even excellent external evidence may be inapplicable to or inappropriate for an individual patient; without current best evidence, practice risks becoming out of date, to the detriment of patient care [1].

Prof. Max Aebi then addressed the changes and challenges of scientific publishing. He examined the history and growth of specialised spine journals since the 1970s. The reasons behind this growth in specialised spine literature are the increase and improvements in spine technology as well as the establishment of specialised basic 
science and clinical research in this field. The pros and cons of the 'impact factor' and 'Eigen factor' as tools to measure the quality of a scientific journal and the challenges and opportunities for journals in the digital age were also discussed.

Dr. Kim Edwards gave a short tutorial on the essential statistics that reviewers need to understand to be able to review the data analyses in a paper. The tutorial used lay language to describe and define some key statistical principles, such as what $p$ values and $95 \%$ confidence intervals really tell us, and examined sample size calculations and power. Risk was also covered, with a comparison of relative risk and odds ratios, and the importance of evaluating absolute risk was demonstrated. She then moved on to look at the steps necessary to judge whether appropriate statistical tests had been used in a study, and provided a brief overview of the information that should be included in each of the different sections of a journal article.

This was followed by a summary of the important aspects of review by Dr. Anne Mannion. She suggested that, when invited to review, the first question to ask yourself is whether you have sufficient expertise to do the job. Then question whether you have any conflicts of interest. Finally, consider whether you have the time to do the review, to the required standard, in a reasonable ( $\max$ 4 weeks) timescale. If yes to all three, your review can begin. She recommended starting with a first read through, to place the paper in one of the three categories: (1) publishable in principle; (2). major flaw(s), addressable in principle; (3) fatally flawed and not publishable. If flawed, the flaw should be documented and substantiated as part of the review. On a second read through, further aspects concerning scientific details, organisation, writing, etc. should be addressed.

Then followed a detailed discussion of the different sections of the journal paper and what information they should contain-importantly, considering what the different sections should be seeking to communicate to the reader. Reference was made to the many reporting guidelines and checklists available, e.g. those listed on http:// www.equator-network.org that can be used for evaluating the quality of reporting for studies of different design. We were reminded of the need for confidentiality. Finally, the principal reasons motivating people to review papers, as revealed by a large-scale survey [2], were presented: these include playing one's part as a member of the academic community, and enjoying seeing other papers and helping to improve them. Almost all researchers surveyed believed that their last paper was improved by the peer-review process. The benefits of peer-reviewing are diverse: from improving your critical thinking, giving and receiving feedback, and gaining insights to improve your future publications; it is considered an essential skill to develop as a researcher.

Prof. Constantin Schizas added some humour to the session with his discussion of 'how not to review a paper', depicting the infamous hostile reviewer as well as reminding us that we do not do it to get rich! The workshop was wound up with a practical exercise looking for errors in a theoretical abstract submitted to the European Spine Journal. Delegates reported finding the session informative and many volunteered to become new reviewers for the journal. Hopefully, it is they who are reviewing your next paper and not Prof. Schizas' hypothetical hostile reviewer!

\section{Conflict of interest None.}

Open Access This article is distributed under the terms of the Creative Commons Attribution License which permits any use, distribution, and reproduction in any medium, provided the original author(s) and the source are credited.

\section{References}

1. Sackett DL, Rosenberg WM, Gray JA, Haynes RB, Richardson WS (1996) Evidence based medicine: what it is and what it isn't. BMJ 312:71-72

2. Campbell R, Heap B, Mulligan A, Hames I (eds) A sense about science, in conjunction with Elsevier, survey. Results from the 2009 peer review survey. http://www.senseaboutscience.org/ pages/peer-review-survey-2009.html 